\title{
28 Research Soure \\ Clock Gene Regulates Kainic Acid-Induced Seizures Through Inhibiting Ferroptosis in Mice
}

Fei Wang

Jinan University College of Pharmacy

\section{Lianxia Guo}

Jinan University College of Pharmacy

Zhengping Wu

Yichun University

Tianpeng Zhang

Guangzhou University of Chinese Medicine

\section{Dong Dong}

Jinan University

Baojian Wu ( $\nabla$ bj.wu@hotmail.com )

Guangzhou University of Chinese Medicine https://orcid.org/0000-0003-4629-5142

\section{Research Article}

Keywords: Clock, epilepsy, ferroptosis, GPX4, PPAR-ץ

Posted Date: November 2nd, 2021

DOI: https://doi.org/10.21203/rs.3.rs-999044/v1

License: (c) (i) This work is licensed under a Creative Commons Attribution 4.0 International License.

Read Full License 


\section{Abstract}

Temporal lobe epilepsy (TLE) is a common and intractable form of epilepsy. There is a strong need to better understand molecular events underlying TLE and to find novel therapeutic agents. Here we aimed to investigate the role of Clock gene and ferroptosis in regulating TLE. TLE model was established by treating mice with kainic acid (KA). Regulatory effects of Clock gene on KA-induced seizures and ferroptosis were evaluated using Clock knockout $\left(\mathrm{Clock}^{-/}\right)$mice. mRNA and protein levels were determined by quantitative real-time PCR and western blotting, respectively. Ferroptosis was assessed by measuring the levels of iron, GSH and ROS. Transcriptional regulation was studied using a combination of luciferase reporter, mobility shift and chromatin immunoprecipitation (ChIP) assays. We found that Clock ablation exacerbated KA-induced seizures in mice, accompanied by enhanced ferroptosis in the hippocampus. Furthermore, Clock ablation reduced the hippocampal expression of GPX4 and PPAR- $\gamma$, two ferroptosis-inhibitory factors, in mice and in N2a cells. Moreover, Clock regulates diurnal expression of GPX4 and PPAR-y in mouse hippocampus and rhythmicity in KA-induced seizures. Consistently, Clock overexpression up-regulated GPX4 and PPAR-y, and protected against ferroptosis in N2a cells. In addition, based on luciferase reporter, mobility shift and ChIP assays, we uncovered that CLOCK protein transactivated Gpx4 and Ppar- $y$ through specific binding to an E-box element in gene promoters. In conclusion, CLOCK protests against KA-induced seizures through promoting expression of GPX4 and PPAR- $\gamma$ and inhibiting ferroptosis.

\section{Introduction}

Epilepsy is a brain disease characterized by unprovoked recurrent seizures, affecting over 70 million people worldwide. Temporal lobe epilepsy (TLE) is a common and intractable form of epilepsy and associated with pathologic changes in hippocampal physiology and morphology'. Approximately $30 \%$ of patients with epilepsy remain intractable to antiseizure drugs, and this subset of patients is usually diagnosed with TLE. Thus, there is a strong need to better understand molecular events underlying TLE and to find novel therapeutic agents. Kainic acid (KA)-induced seizure is regarded as a good model of TLE as the behavioral seizures and neuropathological lesions in the animals are highly similar to those of TLE patients.

Ferroptosis is an iron-dependent, oxidative form of non-apoptotic regulated cell death, characterized by an increase in free iron and accumulation of lipid peroxides. GPX4 (glutathione peroxidase 4) is considered as a key regulator of ferroptosis. It converts the toxic phospholipid hydroperoxides (lipid- $\mathrm{OOH}$ ) to nontoxic phospholipid alcohols (lipid-OH) by utilizing an electron donated by GSH (glutathione). Thus, ferroptosis can be triggered by inhibition of GPX4 or disruption of GSH synthesis ${ }^{7}$. Ferroptosis has been implicated in the development of various pathological conditions such as cancers, neurodegeneration, ischemia reperfusion injury and acute kidney injury. Moreover, various ferroptosis inducers and inhibitors have been shown to modulate disease progression in preclinical models. Therefore, targeting ferroptosis may provide a new avenue for disease management. 
PPARs (peroxisome proliferator-activated receptors) are a family of ligand-responsive nuclear receptors consisting of three members, namely, PPAR-a, PPAR- $\beta$ (or PPAR- $\delta$ ) and PPAR- - . PPARs can be activated by endogenous ligands such as fatty acids and their derivatives. Upon ligand binding, PPARs form heterodimers with retinoid $X$ receptors and bind to a specific DNA response element (PPRE) in promoter to regulate gene transcription. PPAR isoforms show tissue-specific differences in their expression and functions $^{12}$. Although PPAR-y is abundantly present in adipose tissues, it can be found in many other tissues such as the liver and hippocampus. PPAR-y has a critical role in regulating lipid metabolism, insulin sensitivity, tumor cell growth, apoptosis and differentiation. In addition, PPAR- $\gamma$ has the potential to activate defensive mechanisms against lipoperoxidative reactions by controlling the expression of antioxidant enzymes, thereby inhibiting ferroptosis'.

Various aspects of physiology and behaviors in mammals are subjected to circadian rhythms that are driven and maintained by the circadian clock system. Disruption of circadian rhythms is linked to various disorders such as depression, diabetes, cancers, and cardiovascular diseases. At the molecular level, the circadian clock system contains multiple transcriptional-translational feedback loops in which various positive and negative components regulate the expression of clock-controlled genes (CCGs). Clock (circadian locomotor output cycles kaput) is one of core positive regulatory genes in the circadian clock system and is expressed in virtually all tissues. It binds to a specific response element (called "E-box") in the promoters of target genes and activates gene transcription. In addition to regulating circadian rhythms, Clock is also involved in regulation of many other physiological processes including cell cycle, lipid metabolism, glucose metabolism, and immune responses. It is therefore of no surprise that mutations in Clock gene are associated with pathological conditions such as osteoarthritis, atherosclerosis, heart failure, tumorigenesis and bipolar disorder-.

In the present study, we aimed to investigate the role of Clock gene and ferroptosis in regulating TLE on the basis of KA-induced seizure model. Regulatory effects of Clock gene on KA-induced seizures and ferroptosis were evaluated using Clock knockout ( $\left.\mathrm{Clock}^{\prime-}\right)$ mice. mRNA and protein levels were determined by quantitative real-time PCR (qPCR) and western blotting, respectively. Ferroptosis was assessed by measuring the levels of iron, GSH and ROS. Transcriptional regulation was studied using a combination of luciferase reporter, mobility shift and chromatin immunoprecipitation (ChIP) assays. We have demonstrated for the first time that CLOCK protests against KA-induced seizures through promoting expression of GPX4 and PPAR-y and inhibiting ferroptosis.

\section{Materials And Methods}

\subsection{Materials}

KA was purchased from Abcam (Cambridge, MA, USA). Deferoxamine (DFO) and RSL3 were obtained from TaoSu Biochemical Technology (Shanghai, China). Assay kits for iron, malondialdehyde (MDA) and GSH were purchased from Jiancheng Bioengineering Institute (Nanjing, Jiangsu, China). CCK-8 Cell Counting Kit was purchased from TransGen Biotech (Beijing, China). ELISA kit for 4-hydroxynonenal (4- 
HNE) was obtained from Neobioscience (Shenzhen, China). Anti-CLOCK (ab3517) and anti-GAPDH (ab8245) antibodies were obtained from Abcam (Cambridge, MA). Anti-GPX4 (14432-1-AP) and antiPPAR-y (16643-1-AP) antibodies were purchased from Proteintech (Wuhan, China). Gpx4 luciferase reporters (-1974/+38 bp, -1315/+38 bp, -601/+38 bp and a mutated version), Ppar- $y$ luciferase reporters (-1900/+100 bp, -700/+100 bp, -29/+100 bp and a mutated version), pRL-TK, pcDNA3.1-Clock, pcDNA3.1, siClock (siRNA targeting Clock, sequence is shown in Table 1) and siNC (negative control for siRNA, sequence is shown in Table 1) were obtained from Transheep Technologies (Shanghai, China). 
Table 1

Oligonucleotides used in this study.

\begin{tabular}{|c|c|c|}
\hline & Forward (5'to $\left.3^{\prime}\right)$ & Reverse (5'to 3') \\
\hline \multicolumn{3}{|c|}{ Genotyping } \\
\hline Clock $^{-1-}$ & CCATGACTCGCCTTTGTTCCTTACTAG & TGAAGCCATATCCACTGCTGGC \\
\hline Wild-type & CCATGACTCGCCTTTGTTCCTTACTAG & CATGAGCGATGTGATCCTGAAAGC \\
\hline \multicolumn{3}{|l|}{ RT-qPCR } \\
\hline Acsl4 & TCCTCCAAGTAGACCAACCCC & AGTCCAGGGATACGTTCACAC \\
\hline Bid & CCAGTCACGCACCATCTTTG & GTCCATCTCGTTTCTAACCAAGT \\
\hline Dmt1 & TACCTAGACCCAGGAAACATCG & CACTCCAAGTCTCGCTGCAA \\
\hline Dpp4 & ACCGTGGAAGGTTCTTCTGG & CACAAAGAGTAGGACTTGACCC \\
\hline Fth & TGCCTCCTACGTCTATCTGTC & GTCATCACGGTCTGGTTTCTTT \\
\hline Ftl & CCATCTGACCAACCTCCGC & CGCTCAAAGAGATACTCGCC \\
\hline Gpx4 & AGTACAGGGGTTTCGTGTGC & CATGCAGATCGACTAGCTGAG \\
\hline Hspa5 & GACTGCTGAGGCGTATTTGG & AGCATCTTTGGTTGCTTGTCG \\
\hline Lpcat3 & CTACCCGTTGGCTCTGTTTTAC & TGAAGCACGACACATAGCAAG \\
\hline Nfs 1 & TCCTCGGGAGATCATTTTCACT & TCCTTGACCTGTAGAACCTGG \\
\hline Nox 1 & GGGTGAAAGCCATCCTCACA & CACTTCCAAGACTCAGGGGG \\
\hline Nrf2 & TCTTGGAGTAAGTCGAGAAGTGT & GTTGAAACTGAGCGAAAAAGGC \\
\hline Ppar-y & AАCCCACAACCAAATCCACAC & ATCACGGAGAGGTCCACAGA \\
\hline Se & TATCCAATGGGTACTCCGCAG & CCGTTCCCCTTGGTGAATCT \\
\hline Slc7a11 & GGCACCGTCATCGGATCAG & CTCCACAGGCAGACCAGAAAA \\
\hline Tfrc & ATGCCGACAATAACATGAAGGC & ACACGCTTACAATAGCCCAGG \\
\hline Trp53 & CACAGCACATGACGGAGGTC & TCCTTCCACCCGGATAAGATG \\
\hline Ppib & TCCACACCCTTTTCCGGTCC & CAAAAGGAAGACGACGGAGC \\
\hline Decr1 & GGCGGTGCAAGCAGGTTAT & GTGCCCAGTAGCACGATGAC \\
\hline \multicolumn{3}{|l|}{ siRNA } \\
\hline siNC & UUCUCCGAACGUGUCACGUTT & ACGUGACACGUUCGGAGAATT \\
\hline Mut, mu & & \\
\hline
\end{tabular}




\begin{tabular}{|lll|}
\hline & Forward (5'to $\mathbf{3}^{\prime}$ ) & Reverse (5'to $\mathbf{3}^{\text {') }}$ \\
\hline siClock & CCUUAGUAAUGAAGAGUUUTT & AAACUCUUCAUUACUAAGGTT \\
\hline EMSA & & \\
\hline Gpx4 & CTGACCTGGTCACGCGCCCTGCCCGG & CCGGGCAGGGCGCGTGACCAGGTCAG \\
\hline Gpx4 (mut) & CTGACCTCTTCTTGGCCCTTGCCCGG & CCGGGCAAGGGCCAAGAAGAGGTCAG \\
\hline Ppar-y & CTGAGAAGTCACGTTCTGACAG & CTGTCAGAACGTGACTTCTCAG \\
\hline Ppar-y (mut) & CCTAGAAGACCCGTCCCTACAG & CTGTAGGGACGGGTCTTCTAGG \\
\hline ChIP & & \\
\hline Cyp2a5-E-box & GTCTCAGAGTCCAACAGCCTAAAAC & TTTGGTTCCAGCAGGTTC \\
\hline Gpx4-E-box & TCCTTGGGCGGTGAGAAC & GGGGACAAAGAGCCGGTAG \\
\hline Ppar-y-E-box & AGGAGCCTGTGAGACCAA & CCTAGCCAGTCAGATCCAG \\
\hline Distal & AAAGGCAGATTGAAGTTTAG & TTCCTCCTGATAGTAATGGT \\
\hline Mut, mutant. & & \\
\hline
\end{tabular}

\subsection{Animals}

Wild-type C57BL/6 mice were obtained from HFK Bioscience (Beijing, China). Clock ${ }^{-/-}$mice (on a C57BL/ 6 background) have been established and validated in our laboratory. All mice were maintained under a $12 \mathrm{~h}$ light/12 h dark cycle [light on at 7:00 AM ( = zeitgeber time (ZT) 0) and light off at 7:00 PM ( = ZT12)], with free access to food and water. Male mice (8-10 weeks old) were used for experiments. Animal experimental procedures were approved by Institutional Animal Care and Use Committee of Guangzhou University of Chinese Medicine and were performed in accordance with the NIH Guide for the Care and Use of Laboratory Animals. Efforts were made to minimize suffering and the number of mice used in the experiments.

\subsection{KA-induced seizures}

Clock $^{\%-}$ mice and wild-type littermates (WT/ $\mathrm{Clock}^{+/+}$) were treated with KA (i.p., $20 \mathrm{mg} / \mathrm{kg}$ ) to induce acute seizures (status epilepticus). The stages in the status epilepticus were recorded according to the Racine scale as previously described: 0 (no response), 1 (staring and reduced locomotion), 2 (head nodding), 3 (unilateral forelimb clonus), 4 (bilateral forelimb clonus), 5 (rearing and falling), and 6 (status epilepticus and death). Seizure severity was assessed by integrating individual scores per mouse over the duration of the experiment. $24 \mathrm{~h}$ after KA treatment, mice were sacrificed to collect hippocampus. Hippocampal iron, MDA and 4-HNE were measured using their assay kits. In order to collect 
electroencephalogram (EEG), mice were subjected to electrode implantation prior to KA induction as described in our recent publication.

\subsection{H\&E, FJB and TUNEL staining}

Mouse brain tissues were fixed in $4 \%$ paraformaldehyde and embedded in paraffin. 4- $\mu$ m-thick coronal sections from the hippocampus were prepared for conventional H\&E, FJB and TUNEL staining as described $^{30}$. Images were acquired using a Nikon Optiphot fluorescent microscope (Tokyo, Japan). Degenerate/dead neurons, FJB- and TUNEL-positive cells were identified and counted using ImageJ (National Institutes of Health, Bethesda, MD). At least three regions from each section and three sections were imaged for each animal.

\subsection{Immunofluorescence}

Immunofluorescence staining for NeuN and GFAP was performed to examine the neuronal apoptosis and astrocytes in mouse hippocampus as described in our recent publication ${ }^{30}$.

\subsection{Quantitative real time PCR ( $\mathrm{qPCR}$ )}

Total RNA was isolated using RNAiso Plus (Takara, Otsu, Japan), and subjected to reverse transcription with HiScript II Q RT SuperMix (Vazyme, Nanjing, Jiangsu, China). qPCR was performed using SYBR Green Master Mix (Vazyme, Nanjing, China) and determined using previously described reaction conditions. The sequences of all primers are shown in Table 1. The specificity of all primers was checked by BLAST analysis (http://www.ncbi.nlm.nih.gov/BLAST/). Relative gene expression levels were calculated by the equation $2^{-\Delta \Delta C t}$ and normalized to housekeeping gene peptidylprolyl isomerase $B$ (Ppib).

\subsection{Western blotting}

Total protein was extracted with RIPA lysis buffer. Protein concentration was measured by using a BCA kit (Beyotime, Shanghai, China). The protein samples were separated by $10 \%$ SDS-PAGE and then transferred to PVDF membranes. Membranes were blocked with $5 \%$ skimmed milk for $1 \mathrm{~h}$, and incubated with primary antibodies (anti-CLOCK, anti-GPX4, anti-PPAR- $y$ and anti-GAPDH) over night at $4^{\circ} \mathrm{C}$. On the next day, the membranes were incubated with secondary antibody at room temperature for $1 \mathrm{~h}$. Protein bands were imaged by using Omega Lum G imaging system (Aplegen, San Francisco, CA) and quantified by fluorchem 5500 software (Alpha Innotech, San Leandro, CA). GAPDH was used as a loading control.

\subsection{Cell culture and treatment}

Mouse neuroblastoma-2a (N2a) cells were obtained from American Type Culture Collection (Rockville, MA). Cells were cultured in MEM medium supplemented with $10 \%$ fetal bovine serum (Gibco). For gene overexpression experiments, cells were transfected with Clock overexpression plasmid or blank pcDNA3.1 plasmid using the JetPrime transfection kit (Polyplus Transfection, Illkirch, France) according to the manufacturer's protocol. For gene silencing experiments, cells were transfected with siRNA (siClock) or 
siNC using the JetPrime transfection kit. After $24 \mathrm{~h}$ or $48 \mathrm{~h}$, cells were collected for qPCR and Western blotting.

\subsection{Chemical-induced ferroptosis}

N2a cells were treated with RSL3 $(0.6 \mathrm{mM})$ for $24 \mathrm{~h}$ to induce ferroptosis. To measure cellular GSH levels and cell viability, cells were collected and assayed using GSH and CCK8 kits according to the manufacturer's instructions. To measure reactive oxygen species (ROS), cells were incubated with 2',7'dichlorodihydrofluorescein diacetate $(10 \mu \mathrm{M})$. After $1 \mathrm{~h}$, cellular fluorescence signal was detected at excitation (485 nm) and emission (530 nm) wavelengths using a Synergy HT Multi-Mode Microplate Reader (BioTek, Winooski, VT). In order to evaluate the effects of Clock on ferroptosis, cells were pretransfected with overexpression plasmid or siRNA for $48 \mathrm{~h}$ prior to RLS3 induction.

\subsection{Luciferase reporter assays}

N2a cells were co-transfected with luciferase reporter (Gpx4-Luc or Ppar- $y$-Luc), pRL-TK and siClock or pcDNA3.1-Clock or control using the JetPrime transfection kit. After $24 \mathrm{~h}$, cells were lysed in passive lysis buffer. Luciferase activities were assayed using the Dual-Luciferase Reporter Assay System and GloMax 20/20 luminometer (Promega, Madison, WI). Firefly luciferase activity was normalized to renilla luciferase activity, and expressed as relative luciferase unit (RLU).

\subsection{Electrophoretic mobility shift assay (EMSA)}

EMSA was performed using an EMSA kit (Beyotime, Shanghai, China) according the manufacturer's instruction. In brief, nuclear extract from N2a cells were incubated with biotin-labeled probe (unlabeled probe or unlabeled mutated probe was added for competitive experiments) in EMSA binding buffer. The mixture was subjected to $4 \%$ nondenaturing PAGE and transferred onto a Hybond- $\mathrm{N}^{+}$membrane (Amersham, Buckinghamshire, UK). The membrane was incubated with enhanced chemiluminescent and visualized by using Omega Lum G imaging system (Aplegen, San Francisco, CA). Oligonucleotide probes are provided in Table 1.

\subsection{ChIP}

ChIP assays were performed using a SimpleChip plus Enzymatic Chromatin IP kit according to the manufacturer's protocol (Cell Signaling Technology, Beverly, MA). In brief, hippocampal tissues were cross-linked with $1.5 \%$ formaldehyde and terminated by glycine. After digestion with micrococcal nuclease and sonication, the sheared chromatin was immunoprecipitated overnight with anti-CLOCK antibody or normal rabbit IgG (as a control). Immunoprecipitated chromatin was decross-linked at $65^{\circ} \mathrm{C}$ and purified by spin columns. Purified DNAs were used as templates for qPCR with specific primers (Table 1). 


\subsection{Statistical analysis}

All data are presented as mean \pm standard error of the mean (SEM). Statistical differences between two groups were analyzed by Student's t-test. One-way or Two-way ANOVA followed by Bonferroni post hoc test was used for multiple group comparisons. The level of significance was set at $p<0.05(*)$.

\section{Results}

\subsection{Clock ablation exacerbates KA-induced seizures in mice}

Mice were treated with KA to induce acute seizures (status epilepticus). Clock ablation in mice resulted in accelerated progression of behavioral seizure stages, and increased seizure severity and duration (Figure $1 \mathrm{~A} / \mathrm{B} / \mathrm{C})$. Moreover, loss of Clock in mice increased the frequency of seizures after KA induction based on the EEG recordings (Figure 1D). These findings indicated a critical role of Clock gene in KA-induced seizures. The pathological hallmarks of epileptic seizures include neuronal loss and gliosis. Based on H\&E, FJB and TUNEL staining, $\mathrm{Clock}^{-/}$mice showed a higher level of neuron death in the hippocampus (Figure 1E). Supporting this, $\mathrm{Clock}^{-/}$mice had a reduced number of living neurons (indicated by $\mathrm{NeuN}^{+}$ cells) and increased astrogliosis (indicated by GFAP ${ }^{+}$cells) in the hippocampus (Figure 1F). Taken together, these data indicated that Clock ablation exacerbates KA-induced seizures in mice.

\subsection{Clock ablation promotes ferroptosis in mice}

Ferroptosis has been implicated in epileptogenesis'. We showed that the potent iron chelator DFO, an inhibitor of ferroptosis, significantly alleviated KA-induced seizures in wild-type mice (Figure 2A/B), confirming a critical role of ferroptosis in seizure development. We further examined a potential role of ferroptosis in Clock regulation of epileptic seizures. The hippocampal levels of iron, MDA, 4-HNE and Decr1, four indicators of ferroptosis, were measured in $\mathrm{Clock}^{-/}$and control mice after KA induction. We found elevated levels of iron, MDA and 4-HNE, and reduced expression of Decr1 in Clock- mice, suggesting enhanced ferroptosis by Clock loss (Figure 2C). In addition, DFO treatment significantly alleviated KA-induced seizures in $\mathrm{Clock}^{-/}$mice (Figure 2D/E). Taken together, the findings suggested that Clock ablation exacerbates KA-induced seizures probably through promoting ferroptosis in mice.

\subsection{Clock ablation reduces hippocampal expression of GPX4 and PPAR-y, two ferroptosis-inhibitory factors, in mice}

We next investigated the mechanisms by which Clock gene regulates ferroptosis. We analyzed the expression of ferroptosis-related genes (Acs/4, Bid, Dmt1, Dpp4, Fth, Ftl, Gpx4, Hspa5, Lpcat3, Nfs 1, Nox1, Nrf2, Ppar-y, Se, Slc7a11, Tfrc and Trp53) in the hippocampus in Clock/- versus control mice after KA induction. Of these tested genes, Gpx4 and Ppar-y (two ferroptosis-inhibitory genes) mRNAs were 
considerably lower in $\mathrm{Clock}^{-1}$ than in control mice (Figure 3A). Consistently, Clock $\mathrm{K}^{-1}$ mice had lower levels of GPX4 and PPAR-y proteins (Figure 3B). We also assessed the effects of Clock on GPX4 and PPAR-y in mice without KA treatment. Likewise, Clock ablation in mice reduced the mRNA and protein expression of both GPX4 and PPAR-y in the hippocampus (Figure 3C/D). Altogether, Clock ablation reduces hippocampal expression of GPX4 and PPAR-y, two ferroptosis-inhibitory factors, in mice.

\subsection{Clockregulates diurnal expression of GPX4 and PPAR-y in mouse hippocampus and rhythmicity in KA-induced seizures}

Clock is a circadian clock gene, whose expression oscillates with time of the day ${ }^{28}$. We confirmed that CLOCK protein was rhythmically expressed in mouse hippocampus with a nadir at ZT10 in wild-type mice (Figure 4A). Interestingly, GPX4 and PPAR- $y$ expression also varied according to the circadian time in the hippocampus, and their diurnal patterns were similar to that of CLOCK protein (Figure 4A). However, Clock ablation reduced the expression levels of both GPX4 and PPAR- $y$, and blunted their diurnal rhythms (Figure 4A). Therefore, Clock gene had an important role in regulating the rhythmicity in GPX4 and PPAR-Y expression. Previous studies have shown that epileptic seizures in rodents display circadian rhythms, and seizures tend to occur more frequently in the later light than in the dark phase-. Consistently, we found that in wild-type mice the seizures were more severe when KA was injected at ZT10 and less severe when KA was injected at ZT22 (Figure 4B/C). More importantly, the time-dependency of seizure severity was attenuated in $\mathrm{Clock}^{-1}$ mice (Figure 4B/C). Taken together, Clock regulates diurnal expression of GPX4 and PPAR-y in mouse hippocampus and rhythmicity in KA-induced seizures.

\subsection{Clock overexpression up-regulates GPX4 and PPAR-y, and protects against ferroptosis in N2a cells}

RSL3, a known inducer of ferroptosis, was used to induce ferroptosis in N2a cells. As expected, RSL3treated cells showed reduced viability, increased ROS and decreased GSH (Figure 5A/B). Clock silencing (by siRNA) resulted in more extensive ferroptosis as evidenced by lower levels of cell viability and GSH as well as higher ROS accumulation (Figure 5A). In contrast, Clock overexpression led to increased cell viability and GSH, as well as decreased ROS accumulation, suggesting attenuation of RSL3-induced ferroptosis (Figure 5B). Moreover, we found that the mRNAs of Gpx4 and Ppar- $\gamma$ were reduced in Clocksilenced cells, but increased in Clock-overexpressed cells after RSL3 induction (Figure 5C). Likewise, Clock showed a similar effects on the expression of GPX4 and PPAR- $y$ in normal N2a cells (without RSL3 induction). To be specific, Clock silencing led to decreased mRNA and protein expression of both GPX4 and PPAR-y (Figure 6A/B). Overexpression of Clock resulted in elevated mRNA and protein levels of both GPX4 and PPAR-y (Figure 6C/D). Altogether, Clock gene regulates ferroptosis in N2a cells probably through modulating the expression of GPX4 and PPAR-y.

\subsection{CLOCK regulates Gpx4 and Ppar-y transcription via a E- box element}


Since CLOCK protein functions as a transcriptional activator, we next investigated whether it regulates GPX4 and PPAR-y expression via a transcriptional mechanism. Clock overexpression plasmid dosedependently increased the Gpx4 (-1974/+38 bp)-Luc and Ppar-y (-1900/+100 bp)-Luc reporter activities according to luciferase reporter assays (Figure 7A). Consistently, the siRNA targeting Clock gene reduced the Gpx4-Luc and Ppar-Y-Luc reporter activities (Figure 7B). Based on sequence analysis with in silico algorithm (Jasper), we found three E-boxes (putative motif for CLOCK binding and action) in Gpx4 promoter and in Ppar- $y$ promoter (Figure 7C/D). Truncation and mutation experiments demonstrated that -63 bp E-box of Gpx4 and 67 bp E-box of Ppar- $y$ were required for CLOCK actions, while other predicted Eboxes were not (Figure 7C/D). EMSA assays further confirmed direct interactions of CLOCK protein with the identified E-boxes in Gpx4 and Ppar-y (Figure 7E). According to ChIP assays, CLOCK protein can be recruited to Gpx4 and Ppar-y promoter sequences (containing the E-box element) in the hippocampus of wild-type mice (Figure 7F). However, such recruitment was lost in the hippocampus of $\mathrm{Clock}^{-/}$mice (Figure 7F). Taken together, CLOCK protein trans-activated Gpx4 and Ppar- $y$ through specific binding to an E-box element in gene promoters.

\section{Discussion}

In this study, we have defined a protective role of Clock gene in KA-induced seizures in mice (Figure 8). More importantly, we have uncovered that Clock regulates epileptic seizures through inhibiting ferroptosis in the hippocampus. The evidence for the links between Clock, ferroptosis and epileptic seizures is strong. First, DFO, an inhibitor of ferroptosis, significantly alleviated KA-induced seizures in both wild-type and $\mathrm{Clock}^{-/-}$mice (Figure 2). Second, Clock ablation promoted ferroptosis in mice and in N2a cells, whereas Clock overexpression protected against ferroptosis in N2a cells (Figure 5). Third, Clock positively regulated the transcription and expression of both GPX4 and PPAR-y, two ferroptosis-inhibiting factors, through specific binding to an E-box element in target gene promoters (Figure 7). Therefore, Clock gene protests against KA-induced seizures through promoting expression of GPX4 and PPAR-y and inhibiting ferroptosis. Our findings provide increased understanding of the complex pathways for regulation of epileptic seizures by circadian clock.

Previous studies have shown that epileptic seizures in rodents display circadian rhythms, and seizures tend to occur more frequently in the later light than in the dark phase $\mathrm{s}^{35,37}$. It is consistent to find that herein in wild-type mice the seizures were more severe when KA was injected at ZT10 and less severe when KA was injected at ZT22 (Figure 4). Interestingly, the circadian time-dependency of seizure severity was considerably attenuated in $\mathrm{Clock}^{-/}$mice (Figure 4), indicating involvement of Clock in circadian regulation of epileptic seizures. Therefore, the circadian rhythm in seizures appears to arise from the rhythmicity in the extent of ferroptosis caused by circadian expression of GPX4 and PPAR- $y$ that are directly driven by the CLOCK protein. This is supported by the fact that GPX4 and PPAR-y share similar diurnal patterns with that of the CLOCK protein (Figure 4).

Considering that Clock gene has a protective role in epileptic seizures and is down-regulated in epileptic tissues, it is most likely involved in epileptogenesis. We have previously shown that REV-ERBa (a direct 
target of CLOCK) drives the expression of GABA transporters and enhances GABA reuptake, thereby alleviating GABA-mediated inhibition and promoting epileptic seizures ${ }^{30}$. Li et al found that deletion of Clock in pyramidal cells causes seizures during sleep in mice ${ }^{39}$. This phenotype was linked to the alteration of cortical circuits ${ }^{39}$. Therefore, although we have shown that Clock gene protests against KAinduced seizures through inhibiting ferroptosis, there is a possibility that other mechanisms are involved, such as disruption of GABAergic function and alteration of cortical circuits.

KA is an excitatory amino acid. Treatment with KA can cause epileptic seizures in the hippocampus. These seizures propagate to other limbic structures and the induced neuropathlogical changes in the hippocampus are comparable to those of patients with TLE ${ }^{5}$. Thus, the Clock gene may be a promising drug target for treating TLE as small molecules targeting CLOCK such as CLK8 are being identified and synthesized. We found that KA-induced seizures were associated with ferroptosis. This support the notion that ferroptosis contributes to epileptic seizures ${ }^{33,34}$. We showed that the ferroptosis inhibitor DFO reduced (a $\sim 50 \%$ reduction) the severity of seizure in wild-type mice (Figure 2$)$. The reducing effect of DFO was also observed in Clock knockout mice. However, the reduction ( 30\%) is less evident (Figure 2). This was probably because a higher level of ferroptosis caused by Clock deletion was more difficult to be repressed by DFO.

We have identified CLOCK as a positive regulator of PPAR-y based on several lines of evidence. First, Clock ablation reduces hippocampal expression of PPAR-y at both mRNA and protein levels in mice (Figure 3). Second, Clock knockdown reduces, whereas Clock overexpression increases, the expression of PPAR-y in N2a cells (Figure 6). Third, based on a combination of luciferase reporter, mobility shift and ChIP assays, CLOCK protein trans-activated Ppar- $y$ through specific binding to an E-box element in gene promoter (Figure 7). However, Reitz et al found that Ppar- $\gamma$ mRNA expression was up-regulated in the heart in Clock $^{\mathbf{4}}{ }^{19 / \mathbf{\Delta}}{ }^{19}$ (exon 19 deletion of Clock gene and a 51-amino-acid deletion of CLOCK protein) mice, suggesting a negative regulatory effect of Clock on Ppar- $\gamma^{25}$. Although the exact reason for this contradiction was unknown, there was a possibility that the positive versus negative action of CLOCK was tissue dependent. This is because the activity of CLOCK is strongly affected by the cellular microenvironments such as the redox state and the types of cofactors.

It was noteworthy that mouse Ppar- $y$ gene generates two different mRNA transcripts (i.e., Ppar- $y 1$ and Ppar-y2) using two distinct promoters. The primer for qPCR assay of Ppar-y in current study cannot discriminate these two transcripts. Thus, mRNA expression of Ppar- $y$ here measures the total level of Ppar-y1 and Ppar-y2. Although both transcripts are expressed in mouse hippocampus, Ppar- $y 1$ is the dominant form as the expression ratio of Ppar-y 1 over Ppar- $\gamma 2$ is more than 7 and a specific antibody fails to detect Ppar- $\gamma 2$ protein in the hippocampus. Therefore, the western blots most likely detected the Ppar- $y 1$ protein in the hippocampus, and the diurnal pattern may be the authentic rhythm of Ppar- $y 1$ protein (Figure 4).

In summary, we have demonstrated that Clock gene protests against KA-induced seizures through promoting expression of GPX4 and PPAR-y and inhibiting ferroptosis. Our findings enhance a deeper 
understanding of the crosstalk between circadian clock and epileptic seizures. Targeting CLOCK protein may provide a promising approach for management of epileptic seizures.

\section{Abbreviations}

CCGs, clock-controlled genes; ChIP, chromatin immunoprecipitation; Clock, circadian locomotor output cycles kaput; DFO, deferoxamine; EEG, electroencephalogram $\mathbb{E M S A}$, electrophoretic mobility shift assay; Gpx4, glutathione peroxidase 4; GSH, glutathione; HNE, 4-hydroxynonenal; KA, kainic acid; MDA, malondialdehyde; N2a, mouse neuroblastoma-2a; PPARs, peroxisome proliferator-activated receptors; Ppib, peptidylprolyl isomerase B; qPCR, quantitative real-time PCR; TLE, temporal lobe epilepsy; WT, wildtype; ZT, zeitgeber time.

\section{Declarations}

\section{Acknowledgments}

This work was supported by the National Natural Science Foundation of China (grants 81573488 and 81503210)

\section{Ethics approval and consent to participate}

Animal experimental procedures were approved by Institutional Animal Care and Use Committee of Guangzhou University of Chinese Medicine (Appr. date: 2020-11-19; IACUC Issue No: ZYD-2020-111) and were performed in accordance with the NIH Guide for the Care and Use of Laboratory Animals.

\section{Consent for publication}

Not applicable.

\section{Availability of data and material}

The raw data and material supporting the conclusions of this article will be made available by the authors, without undue reservation

\section{Conflicts of interest statement}

The authors have declared that no competing interest exists.

\section{Authorship Contributions}

Participated in research design: Wang, Guo and Wu.

Conducted experiments: Wang, Wu, Zhang, Dong.

Performed data analysis: Wang, Dong, and Wu. 
Wrote or contributed to the writing of the manuscript: Wang, Guo and Wu.

\section{References}

1. Thijs R D, Surges R, O'Brien T J, et al. Epilepsy in adults[J]. The Lancet, 2019, 393(10172): 689-701.

2. Hwang S K, Hirose S. Genetics of temporal lobe epilepsy[J]. Brain and Development, 2012, 34(8): 609-616.

3. Garcia-Finana M, Denby C E, Keller S S, et al. Degree of hippocampal atrophy is related to side of seizure onset in temporal lobe epilepsy[J]. American journal of neuroradiology, 2006, 27(5): 10461052.

4. Laxer K D, Trinka E, Hirsch L J, et al. The consequences of refractory epilepsy and its treatment[J]. Epilepsy \& behavior, 2014, 37: 59-70.

5. Bertoglio D, Amhaoul H, Van Eetveldt A, et al. Kainic acid-induced post-status epilepticus models of temporal lobe epilepsy with diverging seizure phenotype and neuropathology[J]. Frontiers in neurology, 2017, 8: 588.

6. Dixon S J, Lemberg K M, Lamprecht M R, et al. Ferroptosis: an iron-dependent form of nonapoptotic cell death[J]. Cell, 2012, 149(5): 1060-1072.

7. FSeibt T M, Proneth B, Conrad M. Role of GPX4 in ferroptosis and its pharmacological implication[J]. Free Radical Biology and Medicine, 2019, 133: 144-152.

8. Forcina G C, Dixon S J. GPX4 at the crossroads of lipid homeostasis and ferroptosis[J]. Proteomics, 2019, 19(18): 1800311.

9. Fujii J, Homma T, Kobayashi S. Ferroptosis caused by cysteine insufficiency and oxidative insult[J]. Free radical research, 2019: 1-12.

10. Wang J, Liu Y, Wang Y, et al. The Cross-Link between Ferroptosis and Kidney Diseases[J]. Oxidative Medicine and Cellular Longevity, 2021, 2021.

11. Han C, Liu Y, Dai R, et al. Ferroptosis and its potential role in human diseases[J]. Frontiers in pharmacology, 2020, 11: 239.

12. Tyagi S, Gupta P, Saini A S, et al. The peroxisome proliferator-activated receptor: A family of nuclear receptors role in various diseases[J]. Journal of advanced pharmaceutical technology \& research, 2011, 2(4): 236.

13. Kersten S, Desvergne B \& Wahli W. Roles of PPARs in health and disease. Nature. 405, 421-424 (2000).

14. Chan LS, Wells RA. Cross-Talk between PPARs and the Partners of RXR: A Molecular Perspective. PPAR Res. 2009;2009:925309.

15. Stump M, Guo D F, Lu K T, et al. Nervous system expression of PPARy and mutant PPARy has profound effects on metabolic regulation and brain development[J]. Endocrinology, 2016, 157(11): 4266-4275. 
16. Laganà A S, Vitale S G, Nigro A, et al. Pleiotropic actions of peroxisome proliferator-activated receptors (PPARs) in dysregulated metabolic homeostasis, inflammation and cancer: Current evidence and future perspectives[J]. International journal of molecular sciences, 2016, 17(7): 999.

17. Benedusi V, Martorana F, Brambilla L, Maggi A, Rossi D. The peroxisome proliferator-activated receptor Y (PPARY) controls natural protective mechanisms against lipid peroxidation in amyotrophic lateral sclerosis. J Biol Chem. 2012 ;287(43):35899-911.

18. Wilson HE, Stanton DA, Rellick S, Geldenhuys W, Pistilli EE. Breast cancer-associated skeletal muscle mitochondrial dysfunction and lipid accumulation is reversed by PPARG. Am J Physiol Cell Physiol. $2021 ; 320(4)$ :C577-C590.

19. Allada R, Chung BY. Circadian organization of behavior and physiology in Drosophila. Annu Rev Physiol. 2010;72:605-24.

20. Walker W H, Walton J C, DeVries A C, et al. Circadian rhythm disruption and mental health[J]. Translational psychiatry, 2020, 10(1): 1-13.

21. Zhao $\mathrm{M}$, Xing $\mathrm{H}$, Chen $\mathrm{M}$, et al. Circadian clock-controlled drug metabolism and transport[J]. Xenobiotica, 2020, 50(5): 495-505.

22. Li X, Wang S, Yang S, Ying J, Yu H, Yang C, Liu Y, Wang Y, Cheng S, Xiao J, Guo H, Jiang Z, Wang Z. Circadian locomotor output cycles kaput affects the proliferation and migration of breast cancer cells by regulating the expression of E-cadherin via IQ motif containing GTPase activating protein 1 . Oncol Lett. 2018;15(5):7097-7103.

23. Nakashima A, Kawamoto T, Noshiro M, et al. Dec1 and CLOCK regulate Na+/K+-ATPase $\beta 1$ subunit expression and blood pressure[J]. Hypertension, 2018, 72(3): 746-754.

24. Yuan G, Xu L, Cai T, Hua B, Sun N, Yan Z, Lu C, Qian R. Clock mutant promotes osteoarthritis by inhibiting the acetylation of NFKB. Osteoarthritis Cartilage. $2019 ; 27(6): 922-931$.

25. Duong ATH, Reitz CJ, Louth EL, Creighton SEM, Rasouli M, Zwaiman A, Kroetsch JT, Bolz SS, Winters BD, Bailey CDC, Martino TA. The Clock Mechanism Influences Neurobiology and Adaptations to Heart Failure in Clock $\Delta 19 / \Delta 19$ Mice With Implications for Circadian Medicine. Sci Rep. $2019 ; 9(1): 4994$.

26. Jiang Q, Liu H, Wang S, Wang J, Tang Y, He Z, Wu F, Huang Z, Cong X, Ding R, Liang C. Circadian locomotor output cycles kaput accelerates atherosclerotic plaque formation by upregulating plasminogen activator inhibitor-1 expression. Acta Biochim Biophys Sin (Shanghai). $2018 ; 50(9): 869-$ 879.

27. Hashikawa KI, Katamune C, Kusunose N, Matsunaga N, Koyanagi S, Ohdo S. Dysfunction of the circadian transcriptional factor CLOCK in mice resists chemical carcinogen-induced tumorigenesis. Sci Rep. 2017;7(1):9995.

28. Zhao M, Zhao H, Deng J, et al. Role of the CLOCK protein in liver detoxification[J]. British journal of pharmacology, 2019, 176(24): 4639-4652.

29. Ziemann AE, Schnizler MK, Albert GW, Severson MA, Howard MA 3rd, Welsh MJ, et al. Seizure termination by acidosis depends on ASIC1a. Nat Neurosci. 2008;11(7):816-822. 
30. Zhang T, Yu F, Xu H, et al. Dysregulation of REV-ERBa impairs GABAergic function and promotes epileptic seizures in preclinical models[J]. Nature communications, 2021, 12(1): 1-14.

31. Wu D M, Zhang Y T, Lu J, et al. Effects of microRNA-129 and its target gene c-Fos on proliferation and apoptosis of hippocampal neurons in rats with epilepsy via the MAPK signaling pathway[J]. Journal of cellular physiology, 2018, 233(9): 6632-6643.

32. Guo L, Yu F, Zhang T, et al. The clock protein Bmal1 regulates circadian expression and activity of sulfotransferase 1a1 in mice[J]. Drug Metabolism and Disposition, 2018, 46(10): 1403-1410.

33. Ye Q, Zeng C, Le Dong Y W, et al. Inhibition of ferroptosis processes ameliorates cognitive impairment in kainic acid-induced temporal lobe epilepsy in rats[J]. American journal of translational research, 2019, 11(2): 875.

34. Mao X Y, Zhou H H, Jin W L. Ferroptosis induction in pentylenetetrazole kindling and pilocarpineinduced epileptic seizures in mice[J]. Frontiers in neuroscience, 2019, 13: 721.

35. Tchekalarova J, Pechlivanova D, Itzev D, et al. Diurnal rhythms of spontaneous recurrent seizures and behavioral alterations of Wistar and spontaneously hypertensive rats in the kainate model of epilepsy[J]. Epilepsy \& Behavior, 2010, 17(1): 23-32.

36. Pavlova M K, Shea S A, Bromfield E B. Day/night patterns of focal seizures[J]. Epilepsy \& Behavior, 2004, 5(1): 44-49.

37. Fenoglio-Simeone KA, Wilke JC, Milligan HL, Allen CN, Rho JM, Maganti RK. Ketogenic diet treatment abolishes seizure periodicity and improves diurnal rhythmicity in epileptic Kcna1-null mice. Epilepsia. 2009;50(9):2027-34.

38. Sui X, Zhang R, Liu S, et al. RSL3 drives ferroptosis through GPX4 inactivation and ROS production in colorectal cancer[J]. Frontiers in pharmacology, 2018, 9: 1371.

39. Li P, Fu X, Smith NA, Ziobro J, Curiel J, Tenga MJ, Martin B, Freedman S, Cea-Del Rio CA, Oboti L, Tsuchida TN, Oluigbo C, Yaun A, Magge SN, O'Neill B, Kao A, Zelleke TG, Depositario-Cabacar DT, Ghimbovschi S, Knoblach S, Ho CY, Corbin JG, Goodkin HP, Vicini S, Huntsman MM, Gaillard WD, Valdez G, Liu JS. Loss of CLOCK Results in Dysfunction of Brain Circuits Underlying Focal Epilepsy. Neuron. 2017;96(2):387-401.

40. Zheng XY, Zhang HL, Luo Q, Zhu J. Kainic acid-induced neurodegenerative model: potentials and limitations. J Biomed Biotechnol. 2011;2011:457079.

41. Doruk YU, Yarparvar D, Akyel YK, Gul S, Taskin AC, Yilmaz F, Baris I, Ozturk N, Türkay M, Ozturk N, Okyar A, Kavakli IH. A CLOCK-binding small molecule disrupts the interaction between CLOCK and BMAL1 and enhances circadian rhythm amplitude. J Biol Chem. 2020 ;295(11):3518-3531.

42. Winkler $U$, Hirrlinger J. Crosstalk of signaling and metabolism mediated by the NAD+/NADH redox state in brain cells[J]. Neurochemical research, 2015, 40(12): 2394-2401.

43. Zhu Y, Qi C, Korenberg JR, Chen XN, Noya D, Rao MS, Reddy JK. Structural organization of mouse peroxisome proliferator-activated receptor gamma (mPPAR gamma) gene: alternative promoter use and different splicing yield two mPPAR gamma isoforms. Proc Natl Acad Sci U S A. 1995;92(17):7921-5. 
44. Denner LA, Rodriguez-Rivera J, Haidacher SJ, Jahrling JB, Carmical JR, Hernandez CM, Zhao Y, Sadygov RG, Starkey JM, Spratt H, Luxon BA, Wood TG, Dineley KT. Cognitive enhancement with rosiglitazone links the hippocampal PPARyand ERK MAPK signaling pathways. J Neurosci. 2012;32(47):16725-35a.

\section{Figures}

Figure 1

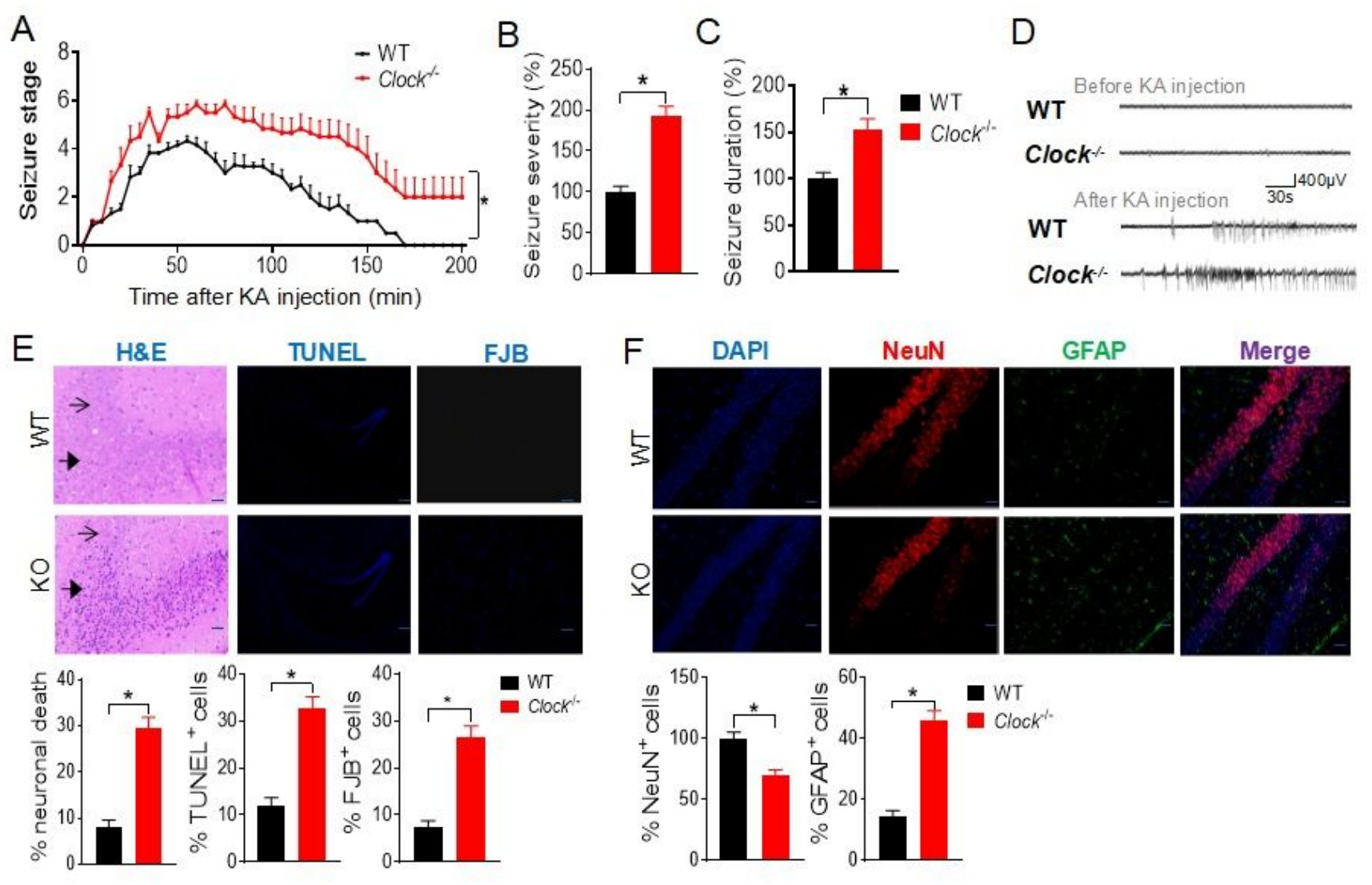

\section{Figure 1}

Clock ablation exacerbates KA-induced seizures in mice. (A) Seizure stages of Clock knockout (Clock-/-) and wild-type (WT) mice injected with KA. Data are mean \pm SEM $(n=6) .{ }^{*} p<0.05$ (two-way ANOVA with Bonferroni post hoc test). (B) Seizure severity of Clock-/- and WT mice injected with KA. Data are mean \pm SEM $(n=6) .{ }^{*} p<0.05$ (t-test). (C) Seizure duration of Clock-/- and WT mice injected with KA. Data are mean \pm SEM $(n=6) .{ }^{*} p<0.05$ (t-test). (D) Representative EEG tracings in Clock-/- and WT mice before (3 h) and after ( $3 \mathrm{~h}) \mathrm{KA}$ injection. (E) Representative images and quantitative analysis of H\&E-stained dead neuron, TUNEL+ cell and FJB+ cell for hippocampus from Clock-/- and WT mice. In H\&E-stained slices, the open arrows show examples of cells couted as alive, and the filled arrows shown examples of cells counted as dead. Scale bar $=50 \mu \mathrm{m}$. Data are mean \pm SEM $(n=6) .{ }^{*} p<0.05$ (t-test). (F) Representative 
images and quantitative analysis of NeuN+ cell and GFAP+ cell for hippocampus from Clock-/- and WT mice. Data are mean \pm SEM $(n=6)$. ${ }^{*} p<0.05$ (t-test). KA, kainic acid.

\section{Figure 2}
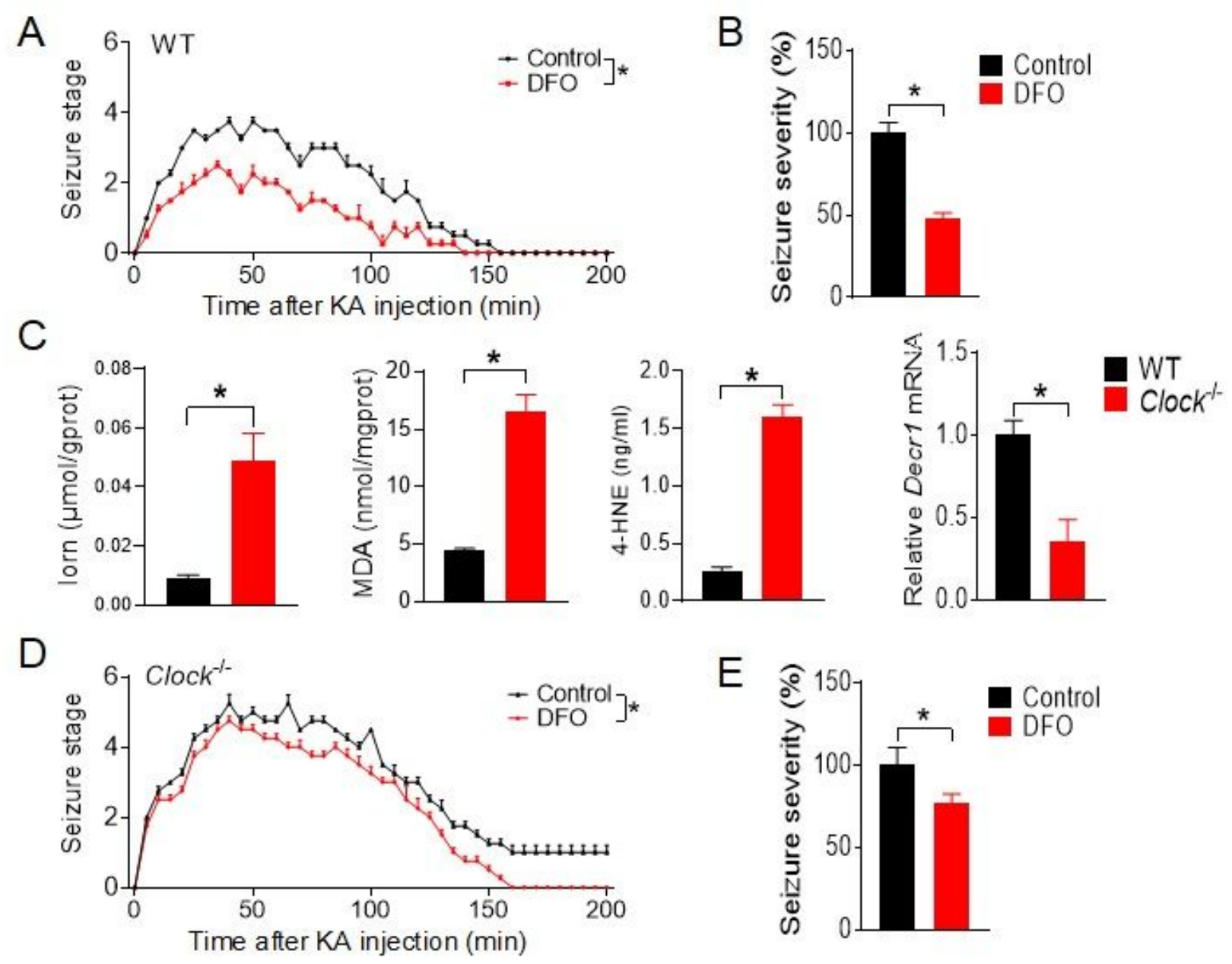

\section{Figure 2}

Clock ablation exacerbates ferroptosis in mice with seizures. (A) Effect of DFO (i.p., $100 \mathrm{mg} / \mathrm{kg}$ ) pretreatment on seizure stages of wild-type (WT) mice injected with KA. Data are mean \pm SEM $(n=6) .{ }^{*} p<$ 0.05 (two-way ANOVA with Bonferroni post hoc test). (B) Effect of DFO pre-treatment on eizure severity of WT mice injected with KA. Data are mean \pm SEM $(n=6) .{ }^{*} p<0.05$ (t-test). (C) Hippocampal iron, MDA and 4-HNE levels as well as Decr1 mRNA expression in Clock knockout (Clock-/-) and WT mice injected with KA. Data are mean \pm SEM $(n=6)$. ${ }^{\star} p<0.05$ (t-test). (D) Effect of DFO pre-treatment on seizure stages of Clock-/- mice injected with KA. Data are mean \pm SEM $(n=6)$. ${ }^{*} p<0.05$ (two-way ANOVA with Bonferroni post hoc test). (E) Effect of DFO pre-treatment on seizure severity of Clock-/- mice injected with KA. Data are mean \pm SEM $(n=6) .{ }^{\star} p<0.05$ (t-test). KA, kainic acid; DFO, Deferoxamine. 


\section{Figure 3}
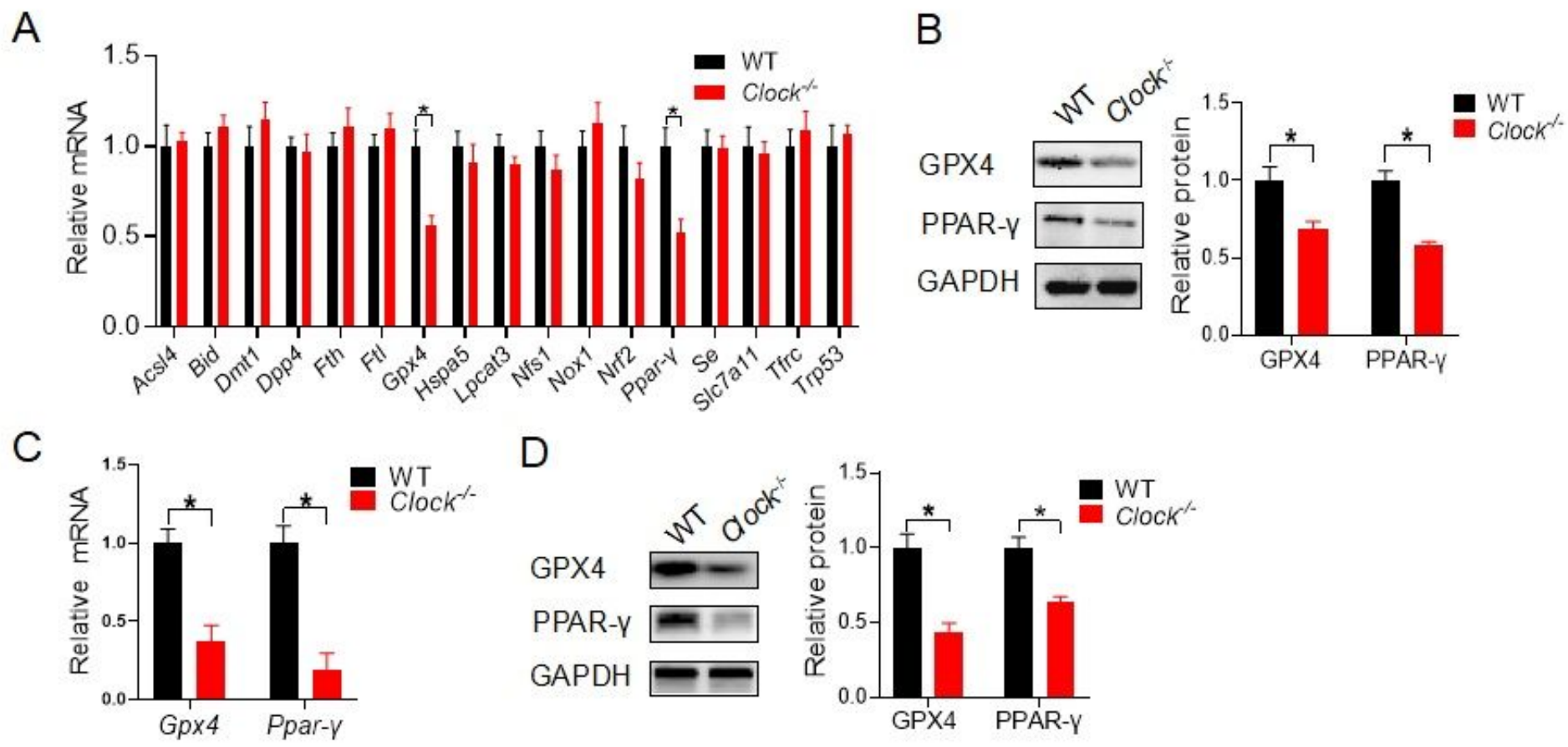

Figure 3

Clock ablation reduces hippocampal levels of GPX4 and PPAR-y in mice. (A) mRNA expression of ferroptosis-related genes in the hippocampus from Clock knockout (Clock-/-) and wild-type (WT) mice injected with KA. (B) Protein expression of GPX4 and PPAR-y in the hippocampus from Clock-/- and WT mice injected with KA. (C) mRNA expression of Gpx4 and Ppar-y in the hippocampus from Clock-/- and WT mice. (D) Protein expression of GPX4 and PPAR-y in the hippocampus from Clock-/- and WT mice. Data are mean \pm SEM $(n=6) .{ }^{*} p<0.05$ (t-test). KA, kainic acid. 
Figure 4

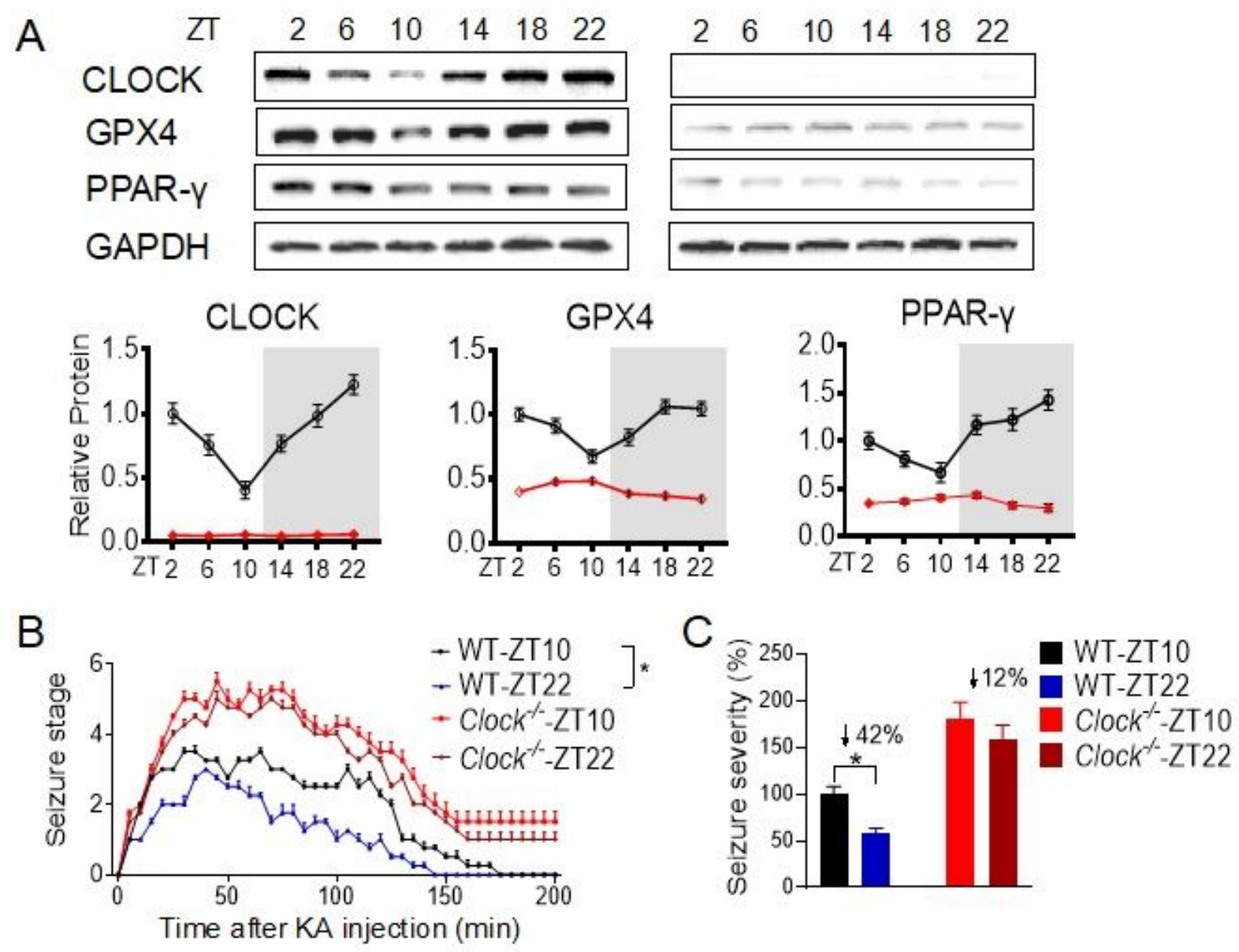

\section{Figure 4}

Clock regulates diurnal expression of GPX4 and PPAR-y in mouse hippocampus and rhythmicity in KAinduced seizures. (A) Diurnal protein expression of hippocampal CLOCK, GPX4 and PPAR- $y$ in Clock knockout (Clock-/-) and wild-type (WT) mice. Data are mean \pm SEM $(n=6)$. * $p<0.05$ (one-way ANOVA with Bonferroni post hoc test). (B) Seizure stages of Clock-/- and WT mice injected with KA at ZT10 and ZT22. Data are mean \pm SEM $(n=6)$. ${ }^{\star} p<0.05$ (one-way ANOVA with Bonferroni post hoc test). (C) Seizure severity of Clock-/- and WT mice injected with KA at ZT10 and ZT22. Data are mean \pm SEM $(n=6)$. * $p<$ 0.05 (t-test). KA, kainic acid. 
Figure 5

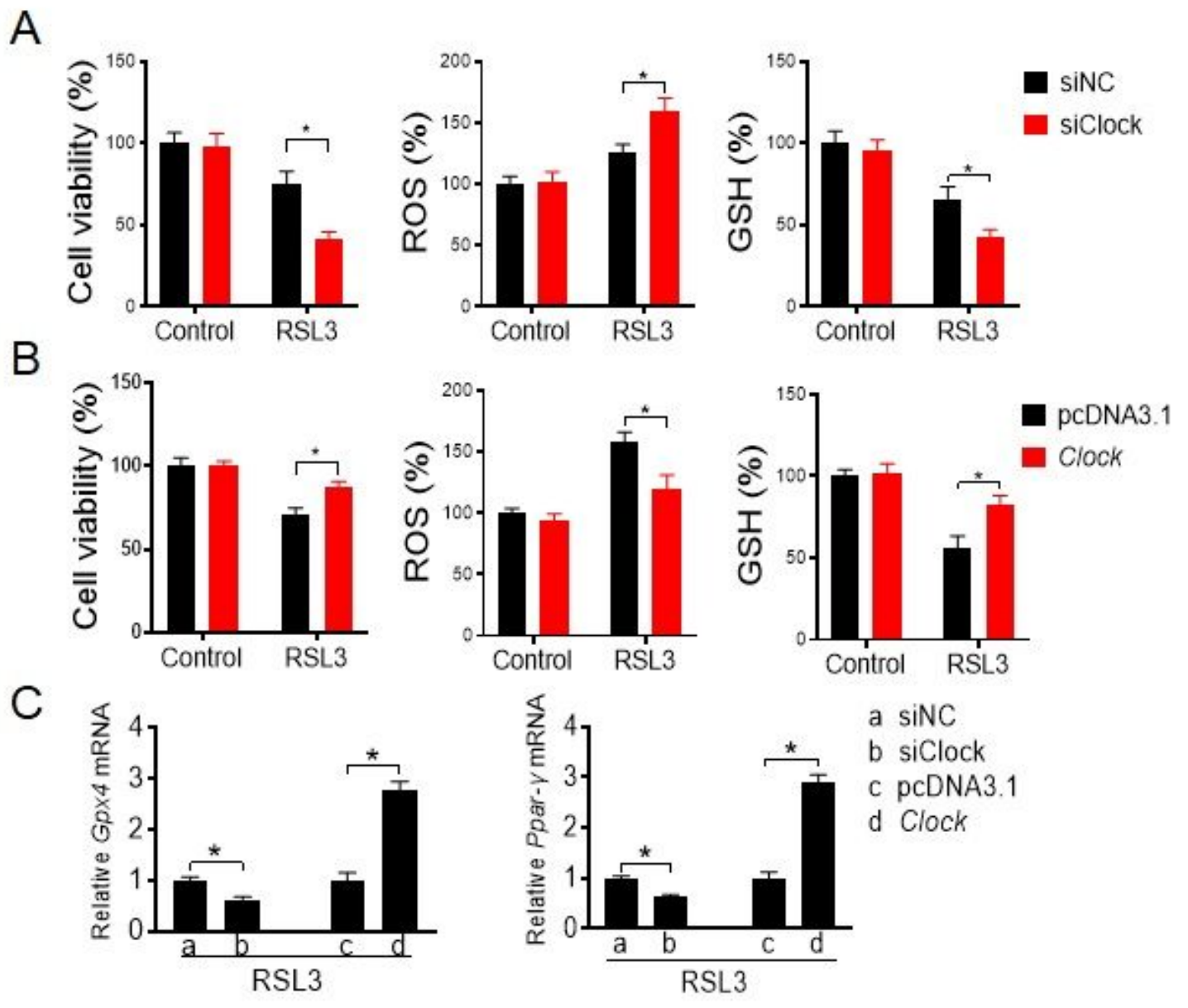

Figure 5

Clock overexpression up-regulates GPX4 and PPAR-y, and protects against ferroptosis in N2a cell . (A) Effects of Clock silencing on cell viability, ROS generation and GSH level in RSL3-treated N2a cells. (B) Effects of Clock overexpression on cell viability, ROS generation and GSH level in RSL3-treated N2a cells. (C) Effects of Clock silencing or overexpression on mRNA expression of Gpx4 and Ppar- $\gamma$ in RSL3-treated N2a cells. Data are mean \pm SEM $(n=6)$. ${ }^{*} p<0.05$ (t-test). 


\section{Figure 6}

A

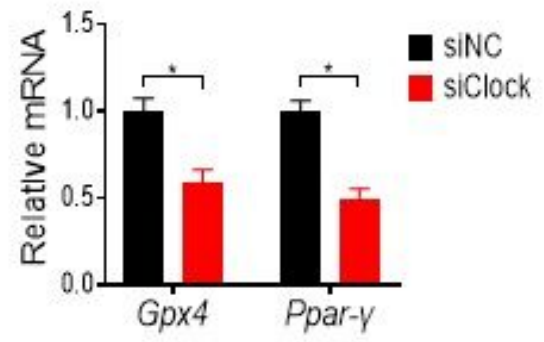

C

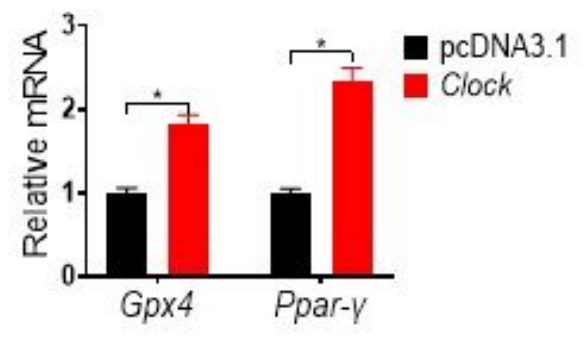

B

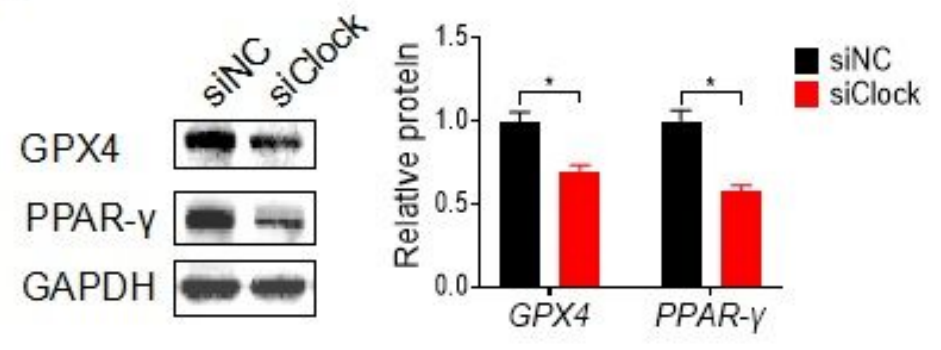

D

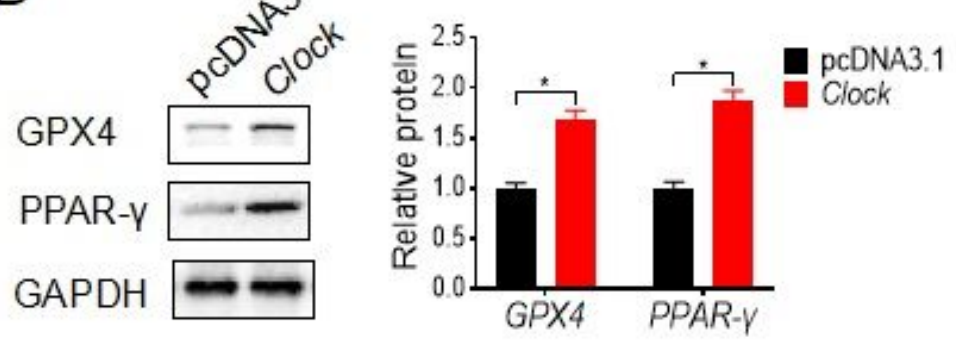

Figure 6

Clock positively regulates the expression of GPX4 and PPAR-y. (A) Effects of Clock silencing on mRNA expression of Gpx4 and Ppar-y in N2a cells. (B) Effects of Clock silencing on protein expression of GPX4 and PPAR-y in N2a cells. (C) Effects of Clock overexpression on mRNA expression of Gpx4 and Ppar-y in N2a cells. (D) Effects of Clock overexpression on protein expression of GPX4 and PPAR- $\gamma$ in N2a cells. Data are mean \pm SEM $(n=6) .{ }^{*} p<0.05$ (t-test). 


\section{Figure 7}

A
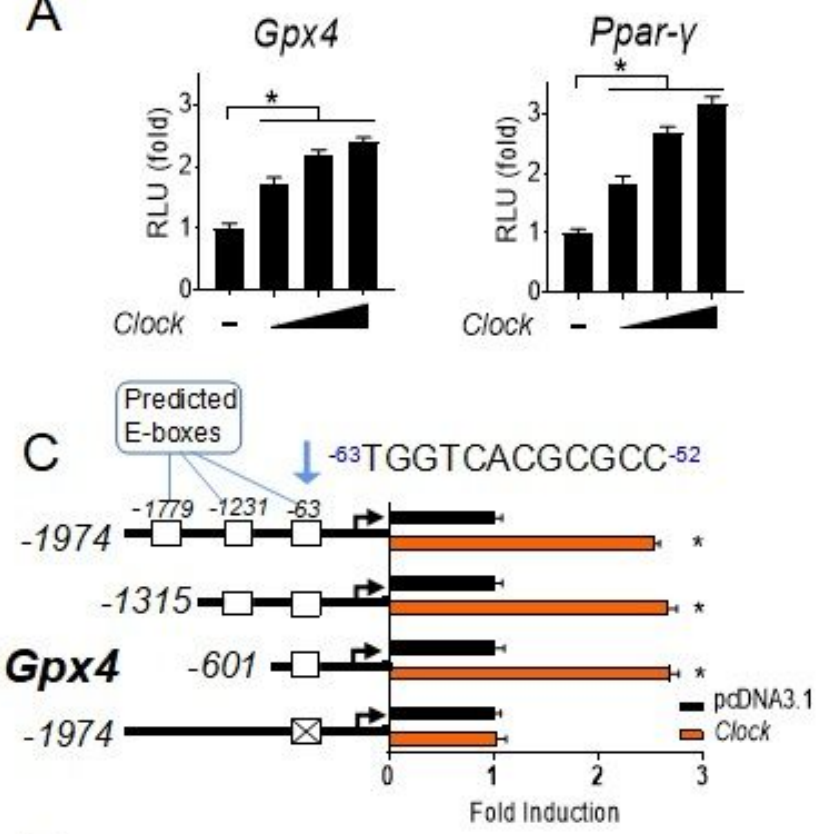

$\mathrm{E}$

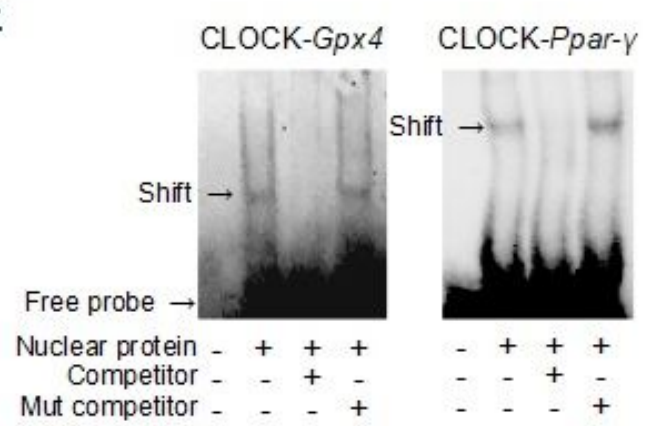

B
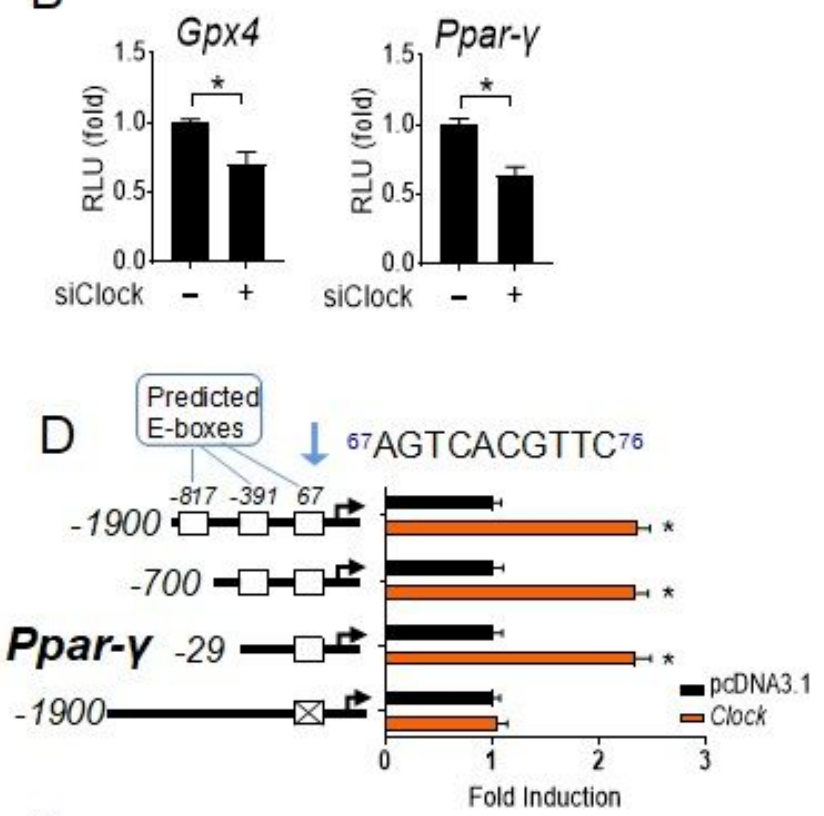

$\mathrm{F}$

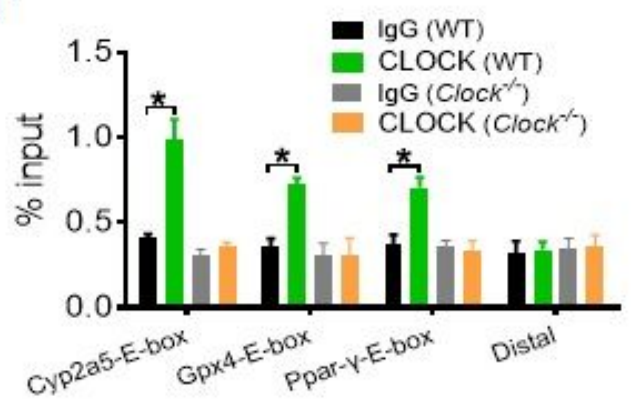

Figure 7

CLOCK regulates Gpx4 and Ppar-y transcription via a E-box element. (A) Clock overexpression increases promoter activities of Gpx4 and Ppar- $\gamma$. (B) Clock silencing reduces promoter activities of Gpx4 and PparY. (C) Effects of Clock on the activities of various versions of Gpx4-Luc promoter in luciferase reporter assays. (D) Effects of Clock on the activities of various versions of Ppar-y-Luc promoter in luciferase reporter assays. (E) EMSA assays showing direct interactions between CLOCK protein and the E-box sites of Gpx4 and Ppar-y. (F) ChIP assays showing recruitment of CLOCK protein to Gpx4 and Ppar-y promoters. In all panels except $E$, data are mean \pm SEM $(n=6)$. ${ }^{*} p<0.05$ (t-test). 
Figure 8

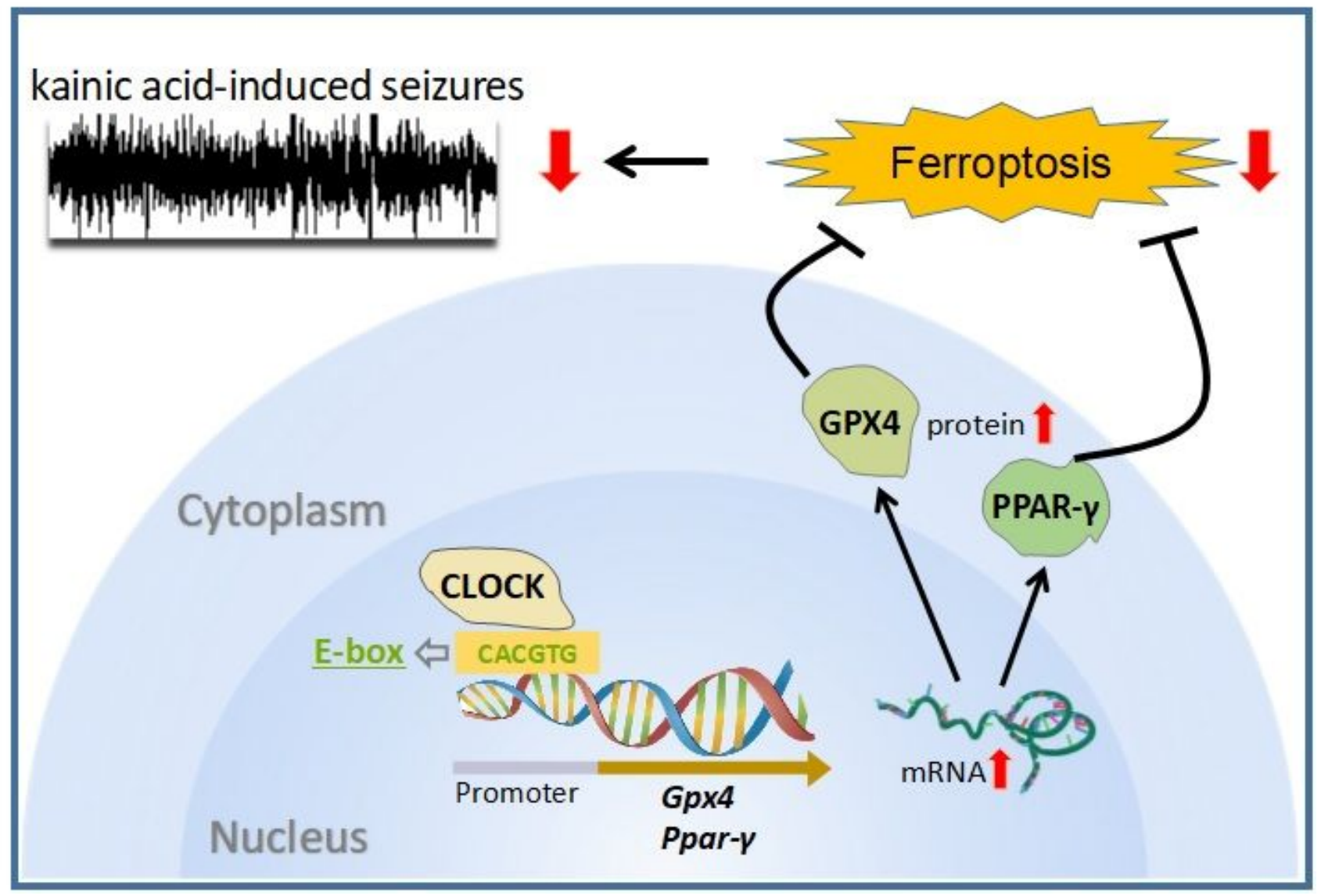

Figure 8

Schematic diagram showing the mechanism for CLOCK regulation of GPX4 and PPAR-y. CLOCK inhibits ferroptosis by promoting the transcription of Gpx4 and Ppar-y via direct binding to an E-box cis-element, thereby protecting against KA-induced epileptic seizures. 